\title{
Intersession delay and avoidance extinction to training and generalization stimuli'
}

\author{
MICHAEL GABRIEL ${ }^{2}$ \\ UNIVERSITY OF WISCONSIN
}

\begin{abstract}
Independent groups of albino rabbits were trained to an avoidance criterion using a 1000 cps pure tone CS. Independent subgroups were extinguished with tones of 1000,2000, or 4000 cps either.5, 12, 24, or $48 \mathrm{~h}$ after training. Resistance to extinction for $1000 \mathrm{cps}$ was least after a.5 h intersession delay (ISD) and greatest after $24 \mathrm{~h}$. ISD did not affect extinction in the $2000 \mathrm{cps}$ groups. For $4000 \mathrm{cps}$, resistance remained low for the three brief ISDs and then increased after $48 \mathrm{~h}$. These results are assumed to reflect a differential rate of increase over time in generalization to other CS values, versus apparatus stimuli.
\end{abstract}

Studies on the relation between intersession delay (ISD) and avoidance transfer (reacquisition) have shown that avoidance responding in reacquisition declines and then rises as ISD increases (Kamin, 1957, 1963; Denny \& Dichtman, 1962; Brush, Meyer, \& Palmer, 1963). The decrease occurs after ISDs ranging from 1 to $4 \mathrm{~h}$, and is called the Kamin effect. The rise is called incubation, and it occurs after $24 \mathrm{~h}$. The present experiment was designed to study incubation under conditions of variation in the test stimulus. Thus, after training with a single tone CS, ISD and CS frequency were varied factorially in an extinction test. An hypothesis presented in Gabriel (1967) suggests that the ISD associated with incubation will increase as the similarity between the training and test CS is decreased.

\section{Subjects and Experimental Design}

The Ss were 96 naive albino rabbits ranging in weight from 3 to 5 pounds and maintained on ad lib food and water. The design was a 3 by 4 factorial with four levels of $\operatorname{ISD}(.5,12,24$, and $48 \mathrm{~h})$ and three levels of test stimulus frequency $(1000,2000$, and $4000 \mathrm{cps})$. The 12 experimental conditions are designated by two hyphenated digits representing an ISD level and a frequency, respectively. The group labels are: .5-1, $.5-2, .5-4,12-1,12-2,12-4,24-1,24-2,24-4,48-1$, 48-2, and 48-4.

\section{Apparafus and Procedure}

The experiment was conducted in a sound insulated light shielded room containing a one way glass through which the $\mathbf{E}$ made his observations. The conditioning apparatus was the rotating cage of Brogden \& Culler (1936). The source for the tone stimulus was a speaker located 12 in. above the top of the rotator. Apparatus for the control of all stimuli was located outside of the experimental room.

Ss were received in groups of 12 and were assigned randomly to the conditions. The CS was a $1000 \mathrm{cps}$ pure tone $3.2 \mathrm{sec}$ in duration and the UCSwas a .2 sec shock which began $3.2 \mathrm{sec}$ following CS onset. CS intensity varied randomly over all trials between five values ranging from 20 to $60 \mathrm{~dB}$ above $.0002 \mathrm{dyne} / \mathrm{cm}^{2}$ in 10 $\mathrm{dB}$ steps. Shock intensity was set at a minimal initial level and was adjusted upward during the first few conditioning trials to provide an optimal cage turning UCR. On all CR trials, the CS was response terminated, and the shock was omitted, by a single switch operation of the $\mathrm{E}$. The ITI varied randomly between 5 and $25 \mathrm{sec}$, with a mean of 15 . Training ( 60 trials per dally session) continued to a criterion of at least nine CRs on a block of 10 trials where blocks do not overlap. A CR was any cage rotation which began during the CS. After criterion attainment, each $\mathrm{S}$ was returned to his home cage for the appropriate ISD. Then each $S$ was carried to the conditioning room and presented with trials with one of the three test tones. Test trials were conducted as training trials except for shock omission in testing. Testing was terminated when at most one CR occurred on any nonoverlapping 10 trial block.

Results

Differences in acquisition in terms of the number of precriterial blocks of 10 trials for the 12 groups were submitted to a 3 by 4 factorial analysis of variance. No significant Fs were obtained in this analysis, suggesting equality of the groups in acquisition blocks.

A 3 by 4 factorial analysis of variance was performed on: (a) the number of blocks of 10 precriterial extinction trials, and (b) the number of precriterial CRs in extinction. Significant results were identical in both analyses and only the results for the (a) measure are presented. A significant $F$ was obtained for stimulus frequency $(p<.01)$ and for the interaction of frequency and ISD $(p<.05)$. The former indicates a decrease for the overall absolute generalization function. The interaction indicates that the relation between ISD and resistance to extinction depends on frequency. Figure 1 presents this interaction with ISD on the abscissa and frequency as the parameter. Within each frequency grouping, $t$ tests for means computed from independent samples were applied to all ISD mean pairs. Significant differences were obtained for the mean of 24-1 vs all other 1 means except 12-1 ( $p<.05$ in each case). The mean for 12-1 does not differ from any of the remaining 1 means. Also, the mean for $48-4$ differs from all remaining (homogeneous) 4 means $(p<.05)$. There are no differences between any of the two means. Thus, resistance to extinction increases after $24 \mathrm{~h}$ and decreases from the $24 \mathrm{~h}$ level after $48 \mathrm{~h}$, for the $1000 \mathrm{cps}$ groups. There is no change in resistance as a function of ISD 


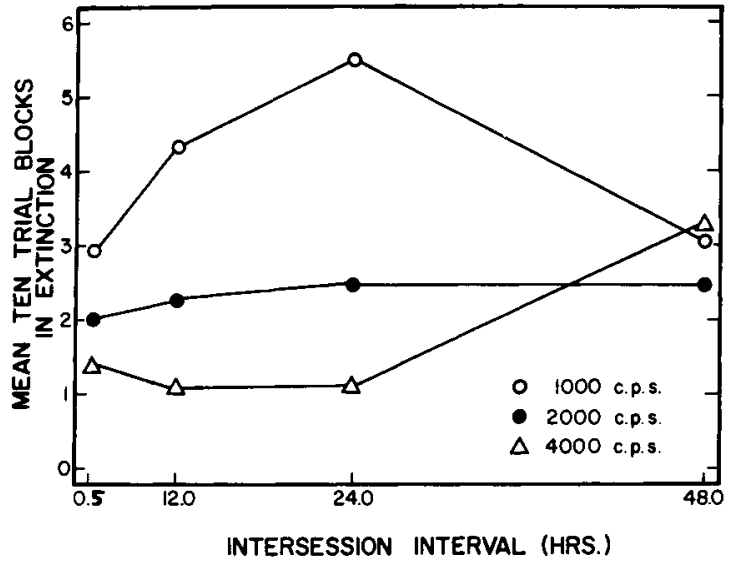

Fig. 1. Mean number of blocks of 10 trials in extinction as a function of ISD, with test stimulus frequency as the parameter.

for the $2000 \mathrm{cps}$ groups, and resistance increases for $4000 \mathrm{cps}$ after $48 \mathrm{~h}$, compared with the briefer ISDs at $4000 \mathrm{cps}$.

\section{Discussion}

Previous findings of a $24 \mathrm{~h}$ increase in test performance are confirmed by differences obtained between the means for the $1000 \mathrm{cps}$ groups. However, the confirmation appears restricted to conditions in which the same stimulus is used for both preISD and postiSD observations. The present data show no differences between the $2000 \mathrm{cps}$ means, and a shift in maximum resistance to extinction toward a higher ISD value for the $4000 \mathrm{cps}$ means, compared with $1000 \mathrm{cps}$. This shift is consistent with the introductory hypothesis. The following explanation for this shift is based on: (a) the demonstrated importance of apparatus stimuli in controlling transfer from classical to avoidance conditioning (McAllister, 1965), and (b) the finding of an increase in responding to a generalization test stimulus, as ISD increases (e.g., McAllister \& McAllister, 1963).

It is assumed that an avoidance CS is always a compound CS. The experimental stimulus (CS) is one element of the compound. The second element consists of tactual, kinesthetic, visual, and auditory stimuli which are present during CS presentation. The latter element is labelled the apparatus complex (AC). The CS can be assumed constant from trial to trial. The AC however, varies such that several different ACs (6-10) occur in compound with the CS over a series of trials. Accord- ingly, the probability of repetition of a single $A C$ is always less than the (perfect) probability of repetition of the CS. Thus for every $x$ trials, $x$ presentations of the CS have occurred together with some number, $q$, of occurrences of each AC, where $q<x$. Thus, the CS undergoes a greater number of repetitions than any $\mathrm{AC}$ in a fixed number of trials. Assuming that repetitions are negatively related to generalization (Thompson, 1958; Hoffeld, 1962), the present shift results might reflect a more rapid increase during the ISD in generalization to a variety of $\mathrm{ACs}$, than to a variety of CSs. Generalization to a variety of ACs is the same as incubation of apparatus fear postulated by McAllister (1965). This incubation potentiates test performance associated with a $24 \mathrm{~h}$ ISD (as in the present Group 24-1). Generalization to a variety of CSs, which requires a longer

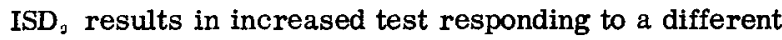
(4000 cps) CS, as in Group 48-1

\section{References}

BROGDEN, W. J., \& CULLER, F. A. A device for the motor conditioning of small animals. Science, 1936, 83, 269-270.

BRUSH, R. F., MEYER, J. S., \& PALMER, M. E. Effects of kind of prior training and intersession interval upon subsequent avoidance learning. J. comp. physiol Psychol., 1963, 56, 539-545.

DENNY, M. R., \& DICHTMAN, R. E. The locus of the maximal "Kamin effect" in rats. J. comp. physiol Psychol., 1962, 55, 1069-1070.

GABRIEL, M. Effects of intersession delay and training level on avoidance extinction and intertrial behavior. Unpublished doctoral thesis, University of Wisconsin, Madison, 1967.

HOFFELD, D. R. Primary stimulus generalization and secondary extinction as a function of strength of conditioning. J. comp. physiol. Psychol, 1962, 55, 27-31.

KAMIN, L. J. The retention of an incompletely learned avoidance response. J. comp. phvsiol. Psychol, 1957, 50, 457-460.

KAMIN, L. J. The retention of an incompletely learned avoidance response: Some further analyses. J. comp. physiol. Psychol., 1963, 56, 713-718.

McALLISTER, W. R., \& McALLISTER, D. E. Increase over time in the stimulus generalization of acquired fear. J. exp. Psychol, $1963,65,576-582$.

McALLISTER, W. R. Variables influencing the conditioning and the measurement of acquired fear. In W. F. Prokasy (Ed.), Classical conditioning. New York: Appleton-Century-Crofts, 1965.

THOMPSON, R. F. Primary stimulus generalization as a function of acquisition level in the cat. J. comp. physiol, Psychol, 1958, 51, 601606.

Notes

1. Supported in part by grants from the National Science Foundation and by the Research Committee of the Graduate School from funds provided by the Wisconsin Alumni Research Foundation. The research is based on a dissertation submitted by the author in partial fulfillment of the requirements for the degree of M.A. at the University of Wisconsin. The author is deeply indebted to W. J. Brogden without whose help and support this research could not have been carried out.

2. Now at Pomona College. 